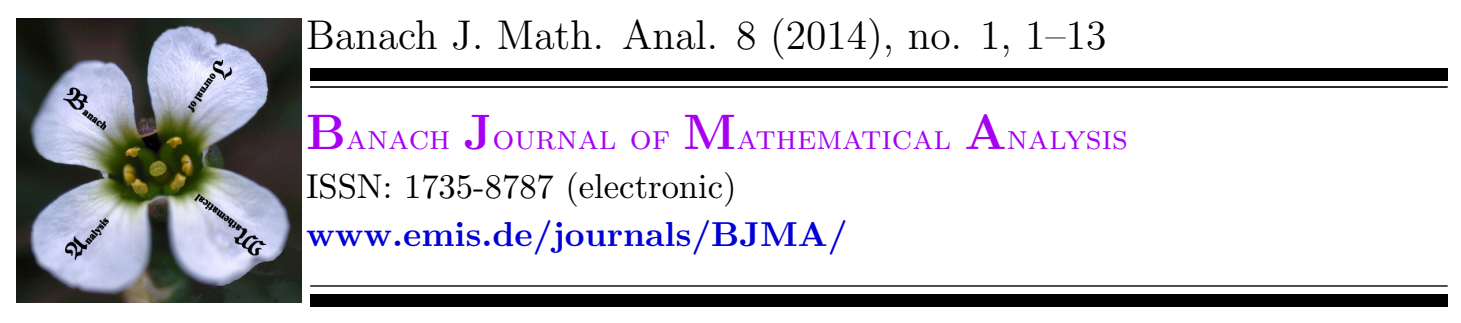

\title{
ON THE EQUIVALENCE BETWEEN SOME MULTIDIMENSIONAL HARDY-TYPE INEQUALITIES
}

\author{
J. A. OGUNTUASE ${ }^{1 *}$, L.-E. PERSSON ${ }^{2}$, N. SAMKO ${ }^{2}$ AND A. SONUBI ${ }^{1}$ \\ Communicated by M. A. Ragusa
}

\begin{abstract}
We prove and discuss some power weighted Hardy-type inequalities on finite and infinite sets. In particular, it is proved that these inequalities are equivalent because they can all be reduced to an elementary inequality, which can be proved by Jensen inequality. Moreover, the corresponding limit (Pólya-Knopp type) inequalities and equivalence theorem are proved. All constants in these inequalities are sharp.
\end{abstract}

\section{INTRODUCTION}

The study of what is today known as the classical Hardy inequality began in 1915 in an attempt by Hardy to find a new and more elementary proof of Hilbert inequality. In this process Hardy [3] in a note published in 1920 announced (without proof) that if $p>1$ and $f$ is a nonnegative $p$-integrable function on $(0, \infty)$, then $f$ is integrable over the interval $(0, x)$ for each positive $x$ and that

$$
\int_{0}^{\infty}\left(\frac{1}{x} \int_{0}^{x} f(t) d t\right)^{p} d x \leq\left(\frac{p}{p-1}\right)^{p} \int_{0}^{\infty} f^{p}(x) d x .
$$

Inequality (1.1), which is usually called the classical Hardy inequality, was proved in 1925 by Hardy in [4]. Nowadays a well-known simple fact is that (1.1) can equivalently via the substitution $f(x)=h\left(x^{1-\frac{1}{p}}\right) x^{-\frac{1}{p}}$, be rewritten in the

Date: Received: 7 November 2012; Accepted: 24 February 2013.

* Corresponding author.

2010 Mathematics Subject Classification. Primary 26D15; Secondary 26A51, 47J20.

Key words and phrases. Inequality, multidimensional Hardy-type inequalities, multidimensional Pólya-Knopp type inequalities, best constant, power weights, convexity. 
form

$$
\int_{0}^{\infty}\left(\frac{1}{x} \int_{0}^{x} h(t) d t\right)^{p} \frac{d x}{x} \leq \int_{0}^{\infty} h^{p}(x) \frac{d x}{x}
$$

and in this form it even holds with equality when $p=1$. Observe that inequality (1.2) can easily be proved by using Jensen inequality and the Fubini theorem. In this form Hardy inequality is a simple consequence of Jensen inequality but it was rather surprising that Hardy did not discovered this fact in the dramatic period of 10 years of research until he finally proved inequality (1.1) in his famous paper [4] from 1925 (see [6, 15, 16]). In fact, Jensen inequality was available since 1905 (see $[8,9]$ ) and he made use of it in many other situations.

In 1965, Godunova [1] remarked this simple direct way of obtaining Hardy inequality via that convexity argument. However, Godunova result in [1] (see also [2]) seems to be fairly little referred to and almost unknown in the western literature. For this reason, the use of this simple convexity argument to obtain Hardy-type inequalities was rediscovered independently by Kaijser et al. [11] in 2002 (cf. also [7]). This was the starting point of many new developments of the subject. After that a great number of results based on this convexity argument have been presented (see e.g. [10, 14, 17, 18, 19, 20, 21, 22, 23, 24] and the PhD thesis [13]) and the references cited therein.

Let us just mention the following special case of a recent result in [24]:

Theorem 1.1. (a): Let $0<l \leq \infty, p \in \mathbb{R}_{+} \backslash\{0\}$ and let $f$ be a nonnegative and measurable function on $(0, l]$. Then

$$
\begin{aligned}
& \int_{0}^{l}\left(\frac{1}{x} \int_{0}^{x} f(y) d y\right)^{p} x^{\epsilon} d x \\
& \leq\left(\frac{p}{p-1-\epsilon}\right)^{p} \int_{0}^{l} f^{p}(x) x^{\epsilon}\left[1-\left(\frac{x}{l}\right)^{\frac{p-\epsilon-1}{p}}\right] d x
\end{aligned}
$$

holds if

$$
\text { (i) } \quad p \geq 1, \epsilon<p-1
$$

or

$$
\text { (ii) } \quad p<0, \epsilon>p-1 \text {. }
$$

(b): Let $0 \leq l<\infty, p \in \mathbb{R}_{+} \backslash\{0\}$ and let $f$ be a nonnegative and measurable function on $[l, \infty]$. Then

$$
\begin{aligned}
& \int_{l}^{\infty}\left(\frac{1}{x} \int_{x}^{\infty} f(y) d y\right)^{p} x^{\epsilon_{0}} d x \\
& \leq\left(\frac{p}{\epsilon_{0}+1-p}\right)^{p} \int_{l}^{\infty} f^{p}(x) x^{\epsilon_{0}}\left[1-\left(\frac{l}{x}\right)^{\frac{\epsilon_{0}+1-p}{p}}\right] d x
\end{aligned}
$$

holds if

$$
\text { (iii) } \quad p \geq 1, \epsilon_{0}>p-1
$$

or

$$
\text { (iv) } \quad p<0, \epsilon_{0}<p-1 \text {. }
$$


(c): All the inequalities above (for all parameters $\epsilon, \epsilon_{0}$ and $p$ ) are equivalent to the basic inequality:

$$
\int_{0}^{l}\left(\frac{1}{x} \int_{0}^{x} g(y) d y\right)^{p} \frac{d x}{x} \leq 1 \int_{0}^{l} g^{p}(x)\left(1-\frac{x}{l}\right) \frac{d x}{x} .
$$

(d): All the inequalities above (i.e. (1.3) - (1.5)) hold in the reversed direction and are sharp.

Remark 1.2. (1) All the inequalities above hold in the reversed direction and are sharp for the case $0<p \leq 1$.

(2) The proof of (1.5) is in fact just a simple application of Jensen inequality and Fubini theorem.

(3) In particular, by using Theorem 1.1 with $l=\infty$ we see that Hardy's first "generalization" from 1928 (see [5]) is not a generalization, in fact it is equivalent to his original inequality (1.1).

In Section 2 of this paper we prove a multidimensional equivalence theorem concerning Hardy-type inequalities, which for $n=1$ contains Theorem 1.1 (see Theorem 2.3). Moreover, in Section 3 we prove a limit result (when $p \rightarrow \infty$ ) of this result, which may be regarded as a equivalence between some multidimensional inequalities of Pólya-Knopp type (see Theorem 3.1).

\section{The main EQUivalenCE THEOREM CONCERNing MUltidimensional HARDY-TYPE INEQUALITIES}

Here and in the sequel we use the boldhead notation $\mathbf{l}$ for $\left(l_{1}, \cdots, l_{n}\right)$. Correspondingly, $(\mathbf{0}, \mathbf{l})$ means the set $\left(0, l_{1}\right) \times\left(0, l_{2}\right) \times \cdots \times\left(0, l_{n}\right), d \mathbf{x}=d x_{1} d x_{2} \cdots d x_{n}$ and $\mathbf{u}^{p}=\left(u_{1} \cdots u_{n}\right)^{p}$. Before we state the main theorem in this section we need the following Lemma of independent interest:

Lemma 2.1. Let $n \in \mathbb{Z}_{+}$and $g$ be a non-negative and measurable function on $(\mathbf{0}, \mathbf{l}), 0<l_{i} \leq \infty, i=1,2, \cdots, n$.

(a): If $p \geq 1$ or $p<0$, then

$$
\begin{aligned}
& \int_{0}^{l_{1}} \cdots \int_{0}^{l_{n}}\left(\frac{1}{x_{1} \cdots x_{n}} \int_{0}^{x_{1}} \cdots \int_{0}^{x_{n}} g(\mathbf{y}) d \mathbf{y}\right)^{p} \frac{d \mathbf{x}}{x_{1} \cdots x_{n}} \\
& \leq 1 . \int_{0}^{l_{1}} \cdots \int_{0}^{l_{n}} g^{p}(\mathbf{y}) \prod_{i=1}^{n}\left(1-\frac{y_{i}}{l_{i}}\right) \frac{d \mathbf{y}}{y_{1} \cdots y_{n}}
\end{aligned}
$$

(in case $p<0$ we assume that $g(\mathbf{x})>0$ for $0<x_{i} \leq b_{i}$ ).

(b): If $0<p \leq 1$, then inequality (2.1) holds in the reversed direction.

(c): The constant $C=1$ in (2.1) is sharp in both cases (a) and (b), respectively.

Remark 2.2. For the case $n=1$ Lemma 2.1 reduces to Theorem 2.1 in [24]. 
Proof. (a) By applying Jensen inequality with the convex function $\phi(u)=u^{p}$, $p \geq 1$ or $p<0$ and Fubini theorem we have that

$$
\begin{aligned}
& \int_{0}^{l_{1}} \cdots \int_{0}^{l_{n}}\left(\frac{1}{x_{1} \cdots x_{n}} \int_{0}^{x_{1}} \cdots \int_{0}^{x_{n}} g(\mathbf{y}) d \mathbf{y}\right)^{p} \frac{d \mathbf{x}}{x_{1} \cdots x_{n}} \\
& \leq \int_{0}^{l_{1}} \cdots \int_{0}^{l_{n}} \frac{1}{x_{1} \cdots x_{n}} \int_{0}^{x_{1}} \cdots \int_{0}^{x_{n}} g^{p}(\mathbf{y}) d \mathbf{y} \frac{d \mathbf{x}}{x_{1} \cdots x_{n}} \\
& =\int_{0}^{l_{1}} \cdots \int_{0}^{l_{n}} g^{p}(\mathbf{y})\left(\int_{y_{1}}^{l_{1}} \cdots \int_{y_{n}}^{l_{n}} \frac{d \mathbf{x}}{x_{1}^{2} \cdots x_{n}^{2}}\right) d \mathbf{y} \\
& =\int_{0}^{l_{1}} \cdots \int_{0}^{l_{n}} g^{p}(\mathbf{y}) \prod_{i=1}^{n}\left(\frac{1}{y_{i}}-\frac{1}{l_{i}}\right) d \mathbf{y} \\
& =\int_{0}^{l_{1}} \cdots \int_{0}^{l_{n}} g^{p}(\mathbf{y}) \prod_{i=1}^{n}\left(1-\frac{y_{i}}{l_{i}}\right) \frac{d \mathbf{y}}{y_{1} \cdots y_{n}}
\end{aligned}
$$

(b) The proof of the case $0<p \leq 1$ is similar to the proof of $(a)$ except that the only inequality (2.2) in the proof holds in the reversed direction.

(c) The proof that the constant $C=1$ in $(a)$ is sharp follows by first considering the case in which the $l_{i}<\infty, i=1,2, \cdots, n$. We assume on the contrary that

$$
\begin{aligned}
& \int_{0}^{l_{1}} \cdots \int_{0}^{l_{n}}\left(\frac{1}{x_{1} \cdots x_{n}} \int_{0}^{x_{1}} \cdots \int_{0}^{x_{n}} g(\mathbf{y}) d \mathbf{y}\right)^{p} \frac{d \mathbf{x}}{x_{1} \cdots x_{n}} \\
& \leq C \int_{0}^{l_{1}} \cdots \int_{0}^{l_{n}} g^{p}(\mathbf{x}) \prod_{i=1}^{n}\left(1-\frac{x_{i}}{l_{i}}\right) \frac{d \mathbf{x}}{x_{1} \cdots x_{n}}
\end{aligned}
$$

for all nonnegative measurable functions $g$ on $(\mathbf{0}, \mathbf{l})$ with some constants $C$, $0<C<1$.

Now suppose that $p \geq 1$ and $\epsilon>0$ and let $g_{\epsilon}(\mathbf{x})=\mathbf{x}^{\epsilon}$. By substituting this function $\mathbf{g}_{\epsilon}(\mathbf{x})$ into inequality (2.3) yields

$$
C \geq(\epsilon p+1)^{n}(\epsilon+1)^{-n p} .
$$

Now, by letting $\epsilon \rightarrow 0_{+}$we obtain that $C \geq 1$, contradicting our assumption that $0<C<1$. This contradiction shows that the best constant in (2.1) is $C=1$. For the case $p<0$ we just use the test function $\mathbf{g}_{\epsilon}(\mathbf{x})=\mathbf{x}^{\epsilon}$ with $\epsilon<0$ and do similar calculations as above to see that the constant $C=1$ is sharp also in this case.

The proof that $C=1$ is sharp also for the case $0<p \leq 1$ is similar and just omitted.

Finally, for the case when one or more of the numbers $l_{i}=\infty$, the sharpness follows by making a limit procedure with the results above in mind and the proof is complete.

We are now ready to state our main result in this section:

Theorem 2.3. Let $0 \leq l_{i} \leq \infty, i=1,2, \cdots, n, n \in \mathbb{Z}_{+}, p \in \mathbb{R}_{+} \backslash\{0\}, \epsilon \in \mathbb{R}$, $\epsilon_{0}=2 p-\epsilon-2$, and let $f$ be a nonnegative function. 
(a): If $f$ is a measurable function on $(\mathbf{0}, \mathbf{l}]$, then

$$
\begin{aligned}
& \int_{0}^{l_{1}} \cdots \int_{0}^{l_{n}}\left(\frac{1}{x_{1} \cdots x_{n}} \int_{0}^{x_{1}} \cdots \int_{0}^{x_{n}} f(\mathbf{y}) d \mathbf{y}\right)^{p} \prod_{i=1}^{n} x_{i}^{\epsilon} d \mathbf{x} \\
\leq & \left(\frac{p}{p-1-\epsilon}\right)^{n p} \int_{0}^{l_{1}} \cdots \int_{0}^{l_{n}} f^{p}(\mathbf{x}) \prod_{i=1}^{n}\left[1-\left(\frac{x_{i}}{l_{i}}\right)^{\frac{p-1-\epsilon}{p}}\right] \\
\times & \prod_{i=1}^{n} x_{i}^{\epsilon} d \mathbf{x}
\end{aligned}
$$

holds for the following cases:

$$
\begin{aligned}
& \text { (i) } \quad p \geq 1, \epsilon<p-1 \\
& \text { (ii) } \quad p<0, \epsilon>p-1 .
\end{aligned}
$$

(b): For the case $0<p \leq 1, \epsilon<p-1$, inequality (2.4) holds in the reversed direction.

(c): If $f$ is a measurable function on $[1, \infty)$, then

$$
\begin{aligned}
& \int_{l_{1}}^{\infty} \cdots \int_{l_{n}}^{\infty}\left(\frac{1}{x_{1} \cdots x_{n}} \int_{x_{1}}^{\infty} \cdots \int_{x_{n}}^{\infty} f(\mathbf{y}) d \mathbf{y}\right)^{p} \prod_{i=1}^{n} x_{i}^{\epsilon_{0}} d \mathbf{x} \\
\leq & \left(\frac{p}{\epsilon_{0}+1-p}\right)^{n p} \int_{l_{1}}^{\infty} \cdots \int_{l_{n}}^{\infty} f^{p}(\mathbf{x}) \prod_{i=1}^{n}\left[1-\left(\frac{l_{i}}{x_{i}}\right)^{\frac{\epsilon_{0}+1-p}{p}}\right] \\
\times & \prod_{i=1}^{n} x_{i}^{\epsilon_{0}} d \mathbf{x}
\end{aligned}
$$

holds for the following cases:

$$
\begin{aligned}
& \text { (iii) } \quad p \geq 1, \epsilon_{0}>p-1 \\
& \text { (iv) } \quad p<0, \epsilon_{0}<p-1 .
\end{aligned}
$$

(d): For the case $0<p \leq 1, \epsilon_{0}>p-1$, inequality (2.5) holds in the reversed direction.

(e): All the inequalities above are sharp.

(f): All the inequalities above are equivalent to the basic inequality (2.1) for $p \geq 1$ and $p<0$ and equivalent to the reverse inequality for $0<p<1$ (and thus equivalent to each other) via suitable substitutions.

Remark 2.4. By setting $n=1$ in Theorem 2.3 we obtain a slightly more precise version of Theorem 2.4 in [24].

Proof. First we consider inequality (2.4) for the case $(i)$ and show that it is equivalent to the inequality (2.1). The crucial step here is to rewrite the inequality (2.4) with $\mathbf{l}_{0}=\left(a_{1}, a_{2}, \cdots, a_{n}\right) \in(\mathbf{0}, \infty]$ and the function $g:(\mathbf{0}, \mathbf{l}) \rightarrow \mathbb{R}$, instead of $\mathbf{l}$ and $f$, respectively, where $a_{i}=l_{i}^{\frac{p}{p-1-\epsilon}}, i=1,2, \cdots, n$, and

$$
f(\mathbf{x})=g\left(x_{1}^{\frac{p-1-\epsilon}{p}}, \cdots, x_{n}^{\frac{p-1-\epsilon}{p}}\right) \prod_{i=1}^{n} x_{i}^{-\frac{\epsilon+1}{p}} .
$$


By using this and suitable substitutions, the left hand side of inequality (2.4) in this setting becomes

$$
\begin{aligned}
& \int_{0}^{a_{1}} \cdots \int_{0}^{a_{n}}\left(\frac{1}{x_{1} \cdots x_{n}} \int_{0}^{x_{1}} \cdots \int_{0}^{x_{n}} g\left(y_{1}^{\frac{p-1-\epsilon}{p}}, \cdots, y_{n}^{\frac{p-1-\epsilon}{p}}\right) \prod_{i=1}^{n} y_{i}^{-\frac{\epsilon+1}{p}} d \mathbf{y}\right)^{p} \prod_{i=1}^{n} x_{i}^{\epsilon} d \mathbf{x} \\
& =\left(\frac{p}{p-1-\epsilon}\right)^{n p} \int_{0}^{a_{1}} \cdots \int_{0}^{a_{n}}\left(\left[\prod_{i=1}^{n} x_{i}^{\frac{p-1-\epsilon}{p}}\right]^{-1} \int_{0}^{x_{1}^{\frac{p-1-\epsilon}{p}}} \cdots \int_{0}^{x_{n}^{p-1-\epsilon}} g(\mathbf{s}) d \mathbf{s}\right)^{-1} \\
& \times\left(\prod_{i=1}^{n} x_{i}\right)^{-1} \mathbf{x} \\
& =\left(\frac{p}{p-1-\epsilon}\right)^{n p+1} \int_{0}^{l_{1}} \cdots \int_{0}^{l_{n}}\left(\left[\prod_{i=1}^{n} y_{i}\right]^{-1} \int_{0}^{y_{1}} \cdots \int_{0}^{y_{n}} g(\mathbf{s}) d \mathbf{s}\right)^{p}\left(\prod_{i=1}^{n} y_{i}\right)^{-1} d \mathbf{y} .
\end{aligned}
$$

Similarly, the right hand side yields

$$
\begin{aligned}
& \left(\frac{p}{p-1-\epsilon}\right)^{n p} \int_{0}^{a_{1}} \cdots \int_{0}^{a_{n}} g^{p}\left(x_{1}^{\frac{p-1-\epsilon}{p}}, \cdots, x_{n}^{\frac{p-1-\epsilon}{p}}\right) \prod_{i=1}^{n}\left[1-\left(\frac{x_{i}}{l_{i}}\right)^{\frac{p-1-\epsilon}{p}}\right] \\
& \times\left(\prod_{i=1}^{n} x_{i}\right)^{-1} d \mathbf{x} \\
& =\left(\frac{p}{p-1-\epsilon}\right)^{n p+1} \int_{0}^{a_{1}^{\frac{p-1-\epsilon}{p}}} \ldots \int_{0}^{a_{n}^{\frac{p-1-\epsilon}{p}}} g^{p}(\mathbf{y}) \prod_{i=1}^{n}\left[1-\left(\frac{y_{i}}{a_{i}^{\frac{p-1-\epsilon}{p}}}\right)\right] \\
& \times\left(\prod_{i=1}^{n} y_{i}\right)^{-1} d \mathbf{y} \\
& =\left(\frac{p}{p-1-\epsilon}\right)^{n p+1} \int_{0}^{l_{1}} \ldots \int_{0}^{l_{n}} g^{p}(\mathbf{y}) \prod_{i=1}^{n}\left[1-\left(\frac{y_{i}}{l_{i}}\right)\right]\left(\prod_{i=1}^{n} y_{i}\right)^{-1} d \mathbf{y} .
\end{aligned}
$$

Since we have only equalities in the calculations above we conclude that (2.1) and (2.4) are equivalent and, hence, by Lemma 2.1 (a), the proof of case $(i)$ follows.

For the case $(i i)$ all calculations above hold true and, hence, according to Lemma 2.1, inequality (2.1) also holds in this case and, thus, $(a)$ is proved also for the case $(i i)$.

For the case $0<p \leq 1, \epsilon<p-1$, all the calculations above are still valid and so (2.1) holds in the reversed direction according to Lemma 2.1. Hence, $(b)$ is proved.

For the proof of $(c)$ we consider inequality (2.4) with $f(\mathbf{x})$ replaced by $f\left(\frac{1}{x_{1}}, \frac{1}{x_{2}}, \cdots, \frac{1}{x_{n}}\right)$, with $\epsilon$ replaced by $\epsilon_{0}$ to be chosen later on and $\mathbf{l}$ replaced by $\mathrm{l}_{0}=\left(\frac{1}{l_{1}}, \frac{1}{l_{2}}, \cdots, \frac{1}{l_{n}}\right)$ to obtain that 


$$
\begin{aligned}
& \left.\int_{0}^{\frac{1}{l_{1}}} \cdots \int_{0}^{\frac{1}{l_{n}}}\left(\frac{1}{x_{1} \cdots x_{n}} \int_{0}^{x_{1}} \cdots \int_{0}^{x_{n}} f\left(\frac{1}{y_{1}}, \cdots, \frac{1}{y_{n}}\right)\right) d \mathbf{y}\right)^{p} \\
& \times \prod_{i=1}^{n} x_{i}^{\epsilon_{0}} d \mathbf{x} \\
& \leq\left(\frac{p}{p-1-\epsilon_{0}}\right)^{n p} \int_{0}^{\frac{1}{l_{1}}} \cdots \int_{0}^{\frac{1}{l_{n}}} f^{p}\left(\frac{1}{x_{1}}, \cdots, \frac{1}{x_{n}}\right) \\
& \times \prod_{i=1}^{n}\left[1-\left(x_{i} l_{i}\right)^{\frac{p-1-\epsilon_{0}}{p}}\right] \prod_{i=1}^{n} x_{i}^{\epsilon_{0}} d \mathbf{x}
\end{aligned}
$$

By making use of suitable substitution and putting $\frac{f(\mathbf{s})}{s_{1}^{2} \cdots s_{n}^{2}}=g(\mathbf{s})$ we find that the left hand side (LHS) of (2.6) yields

$$
\begin{aligned}
L H S & =\int_{0}^{\frac{1}{l_{1}}} \cdots \int_{0}^{\frac{1}{l_{n}}}\left(\frac{1}{x_{1} \cdots x_{n}} \int_{\frac{1}{x_{1}}}^{\infty} \cdots \int_{\frac{1}{x_{n}}}^{\infty} \frac{f(\mathbf{s})}{s_{1}^{2} \cdots s_{n}^{2}} d \mathbf{s}\right)^{p} \prod_{i=1}^{n} x_{i}^{\epsilon_{0}} d \mathbf{x} \\
& =\int_{l_{1}}^{\infty} \cdots \int_{l_{n}}^{\infty}\left(y_{1} \cdots y_{n} \int_{y_{1}}^{\infty} \cdots \int_{y_{n}}^{\infty} \frac{f(\mathbf{s})}{s_{1}^{2} \cdots s_{n}^{2}} d \mathbf{s}\right)^{p} \prod_{i=1}^{n} y_{i}^{-\epsilon_{0}-2} d \mathbf{y} \\
& =\int_{l_{1}}^{\infty} \cdots \int_{l_{n}}^{\infty}\left(\frac{1}{y_{1} \cdots y_{n}} \int_{y_{1}}^{\infty} \cdots \int_{y_{n}}^{\infty} \frac{f(\mathbf{s})}{s_{1}^{2} \cdots s_{n}^{2}} d \mathbf{s}\right)^{p} \prod_{i=1}^{n} y_{i}^{2 p-\epsilon_{0}-2} d \mathbf{y} \\
& =\int_{l_{1}}^{\infty} \cdots \int_{l_{n}}^{\infty}\left(\frac{1}{y_{1} \cdots y_{n}} \int_{y_{1}}^{\infty} \cdots \int_{y_{n}}^{\infty} g(\mathbf{s}) d \mathbf{s}\right)^{p} \prod_{i=1}^{n} y_{i}^{2 p-\epsilon_{0}-2} d \mathbf{y} .
\end{aligned}
$$

Moreover, the right hand side (RHS) of (2.6) in this setting can with similar substitutions be rewritten as follows:

$$
\begin{aligned}
R H S & =\left(\frac{p}{p-1-\epsilon_{0}}\right)^{n p} \int_{l_{1}}^{\infty} \cdots \int_{l_{n}}^{\infty} f^{p}(\mathbf{y}) \prod_{i=1}^{n}\left[1-\left(\frac{l_{i}}{y_{i}}\right)^{\frac{p-\epsilon_{0}-1}{p}}\right] \\
& \times \prod_{i=1}^{n} y_{i}^{-\epsilon_{0}} \prod_{i=1}^{n} y_{i}^{-2} d \mathbf{y} \\
& =\left(\frac{p}{p-1-\epsilon_{0}}\right)^{n p} \int_{l_{1}}^{\infty} \cdots \int_{l_{n}}^{\infty} g^{p}(\mathbf{y}) \prod_{i=1}^{n}\left[1-\left(\frac{l_{i}}{y_{i}}\right)^{\frac{p-\epsilon_{0}-1}{p}}\right] \\
& \times \prod_{i=1}^{n} y_{i}^{2 p-\epsilon_{0}-2} d \mathbf{y} .
\end{aligned}
$$

By replacing $2 p-\epsilon_{0}-2$ by $\epsilon$ and $g$ by $f$ we obtain that $\epsilon_{0}=2 p-\epsilon-2$, so that $p-1-\epsilon_{0}=\epsilon+1-p$. 
Then, from (2.6), (2.7) and (2.8) we obtain that

$$
\begin{aligned}
& \int_{l_{1}}^{\infty} \cdots \int_{l_{n}}^{\infty}\left(\frac{1}{x_{1} \cdots x_{n}} \int_{x_{1}}^{\infty} \cdots \int_{x_{n}}^{\infty} f(\mathbf{s}) d \mathbf{s}\right)^{p} \prod_{i=1}^{n} x_{i}^{\epsilon} d \mathbf{y} \\
& \leq\left(\frac{p}{\epsilon+1-p}\right)^{n p} \int_{l_{1}}^{\infty} \cdots \int_{l_{n}}^{\infty} f^{p}(\mathbf{x}) \prod_{i=1}^{n}\left[1-\left(\frac{l_{i}}{x_{i}}\right)^{\frac{\epsilon+1-p}{p}}\right] \prod_{i=1}^{n} x_{i}^{\epsilon} d \mathbf{x} .
\end{aligned}
$$

Furthermore,

$$
\epsilon_{0}<p-1 \Longleftrightarrow 2 p-\epsilon-2<p-1 \Longleftrightarrow \epsilon>p-1 \text {. }
$$

Thus $(c)$ with the conditions $(i i i)$ and $(i v)$ is equivalent to $(a)$ with the conditions $(i)$ and $(i i)$, respectively and, hence, also $(c)$ is proved.

For the proof of $(d)$ we just note that the above calculations in this case hold too and that the only inequality from Lemma 2.1 holds in the reversed direction.

Finally, we note that the above proof consists of suitable substitutions and equalities to reduce all inequalities to the sharp inequality (2.1) and we obtain a proof also of the statements $(e)$ and $(f)$ according to Lemma 2.1. The proof is complete.

\section{A Limit RESUlt AND CONCLUDing REMARKS AND EXAMPLES}

Our main result in this Section is the following (limit) equivalence theorem between some multidimensional Pólya-Knopp type inequalities.

Theorem 3.1. Let $0 \leq l_{i} \leq \infty, n=1,2, \cdots, n, n \in \mathbb{Z}_{+}, \epsilon \in \mathbb{R}_{+} \backslash\{0\}, \epsilon_{0}=-2-\epsilon$ and let $f$ be a nonnegative function.

(a): If $f$ is a measurable function on $(\mathbf{0}, \mathbf{l}]$, then

$$
\begin{aligned}
& \int_{0}^{l_{1}} \cdots \int_{0}^{l_{n}} \exp \left(\frac{1}{x_{1} \cdots x_{n}} \int_{0}^{x_{1}} \cdots \int_{0}^{x_{n}} \ln f(\mathbf{y}) d \mathbf{y}\right) \prod_{i=1}^{n} x_{i}^{\epsilon} d \mathbf{x} \\
& \leq e^{(1+\epsilon) n} \int_{0}^{l_{1}} \cdots \int_{0}^{l_{n}} f(\mathbf{x}) \prod_{i=1}^{n}\left(1-\frac{x_{i}}{l_{i}}\right) \prod_{i=1}^{n} x_{i}^{\epsilon} d \mathbf{x} .
\end{aligned}
$$

(b): If $f$ is a measurable function on $[1, \infty)$, then

$$
\begin{aligned}
& \int_{l_{1}}^{\infty} \cdots \int_{l_{n}}^{\infty} \exp \left(x_{1} \cdots x_{n} \int_{x_{1}}^{\infty} \cdots \int_{x_{n}}^{\infty} \ln f(\mathbf{y})\left(\prod_{i=1}^{n} y_{i}^{2}\right)^{-1} d \mathbf{y}\right) \\
& \times \prod_{i=1}^{n} x_{i}^{\epsilon_{0}} d \mathbf{x} \\
& \leq e^{-\left(1+\epsilon_{0}\right) n} \int_{l_{1}}^{\infty} \cdots \int_{l_{n}}^{\infty} f(\mathbf{x}) \prod_{i=1}^{n}\left(1-\frac{l_{i}}{x_{i}}\right) \prod_{i=1}^{n} x_{i}^{\epsilon_{0}} d \mathbf{x} .
\end{aligned}
$$

(c): The inequalities in $(a)$ and $(b)$ are equivalent.

(d): The constants in both (3.1) and (3.2) are sharp. 
Proof. First we replace $f(\mathbf{x})$ by $(f(\mathbf{x}))^{\frac{1}{p}}$ in (2.4) for the case $p>1$ and we obtain that

$$
\begin{aligned}
& \int_{0}^{l_{1}} \cdots \int_{0}^{l_{n}}\left(\frac{1}{x_{1} \cdots x_{n}} \int_{0}^{x_{1}} \cdots \int_{0}^{x_{n}}(f(\mathbf{y}))^{\frac{1}{p}} d \mathbf{y}\right)^{p} \prod_{i=1}^{n} x_{i}^{\epsilon} d \mathbf{x} \\
& \leq\left(\frac{p}{p-1-\epsilon}\right)^{n p} \int_{0}^{l_{1}} \cdots \int_{0}^{l_{n}} f(\mathbf{x}) \prod_{i=1}^{n}\left[1-\left(\frac{x_{i}}{l_{i}}\right)^{\frac{p-1-\epsilon}{p}}\right] \prod_{i=1}^{n} x_{i}^{\epsilon} d \mathbf{x} .
\end{aligned}
$$

By letting $p \rightarrow \infty$ it yields that

$$
\left(\frac{p}{p-1-\epsilon}\right)^{n p} \rightarrow e^{(1+\epsilon) n} \text { and } \frac{p-1-\epsilon}{p} \rightarrow 1
$$

and

$$
\left(\frac{1}{x_{1} \cdots x_{n}} \int_{0}^{x_{1}} \cdots \int_{0}^{x_{n}}(f(\mathbf{y}))^{\frac{1}{p}} d \mathbf{y}\right)^{p} \rightarrow \exp \frac{1}{x_{1} \cdots x_{n}} \int_{0}^{x_{1}} \cdots \int_{0}^{x_{n}} \ln f(\mathbf{y}) d \mathbf{y}
$$

(the scale of power means $\mathcal{P}_{\alpha}, \alpha=\frac{1}{p}$, converges to the geometric mean $\mathcal{P}_{0}$ when $\alpha \rightarrow 0)$. The proof of (3.1) follows by just combining (3.3) - (3.5).

For the proof of $(b)$ we first use the substitutions $y_{i}=\frac{1}{z_{i}}, i=1, \cdots, n$, in (3.1) so that $d \mathbf{y}=-\left(\prod_{i=1}^{n} z_{i}^{2}\right)^{-1} d \mathbf{z}$ and find that the left hand side (LHS) in (3.1) is equal to

$$
\begin{aligned}
& \text { LHS }=\int_{0}^{l_{1}} \cdots \int_{0}^{l_{n}} \exp \left(\frac{1}{x_{1} \cdots x_{n}} \int_{\frac{1}{x_{1}}}^{\infty} \cdots \int_{\frac{1}{x_{n}}}^{\infty} \ln f\left(\frac{1}{z_{1}}, \cdots, \frac{1}{z_{n}}\right)\left(\prod_{i=1}^{n} z_{i}^{2}\right)^{-1} d \mathbf{z}\right) \\
& \times \prod_{i=1}^{n} x_{i}^{\epsilon} d \mathbf{x} .
\end{aligned}
$$

We replace the function $f\left(\frac{1}{z_{1}}, \cdots, \frac{1}{z_{n}}\right)$ by $g\left(z_{1}, \cdots, z_{n}\right)$ and make the substitutions $y_{i}=\frac{1}{x_{i}}, i=1,2, \cdots, n$, to find that

$L H S=\int_{\frac{1}{l_{1}}}^{\infty} \cdots \int_{\frac{1}{l_{n}}}^{\infty} \exp \left(y_{1} \cdots y_{n} \int_{y_{1}}^{\infty} \cdots \int_{y_{n}}^{\infty} \ln g(\mathbf{z})\left(\prod_{i=1}^{n} z_{i}^{2}\right)^{-1} d \mathbf{z}\right) \prod_{i=1}^{n} y_{i}^{-2-\epsilon} d \mathbf{y}$.

By making the similar substitutions and manipulations to the right hand side (RHS) of (3.1) we find that

$$
(R H S) e^{-(1+\epsilon) n}=\int_{\frac{1}{l_{1}}}^{\infty} \cdots \int_{\frac{1}{l_{n}}}^{\infty} g\left(y_{1}, \cdots, y_{n}\right) \prod_{i=1}^{n}\left(1-\frac{1}{y_{i} l_{i}}\right) \prod_{i=1}^{n} y_{i}^{-2-\epsilon} d \mathbf{y}
$$

By replacing $l_{i}$ by $\frac{1}{l_{i}}, g(\mathbf{x})$ by $f(\mathbf{x})$ and combining (3.6) and (3.7) we obtain (3.2) so also (3.2) is proved.

The sharpness of the constant $e^{(1+\epsilon) n}$ in (3.1) is just a consequence of the sharpness of the inequalities (2.4) for all $p>1$ and a continuity argument. Since all calculations above only consist of equalities, it is clear that also the constant 
$e^{-\left(1+\epsilon_{0}\right) n}$ in (3.2) is sharp and that in fact the inequalities (3.1) and (3.2) are equivalent. The proof is complete.

Example 3.2. Let $\epsilon=0$ and all $l_{i}^{\prime} s=\infty$. Then the inequality (3.1) reads:

$$
\begin{aligned}
& \int_{0}^{\infty} \cdots \int_{0}^{\infty} \exp \left(\frac{1}{x_{1} \cdots x_{n}} \int_{0}^{x_{1}} \cdots \int_{0}^{x_{n}} \ln f(\mathbf{y}) d \mathbf{y}\right) d \mathbf{x} \\
& \leq e^{n} \int_{0}^{\infty} \cdots \int_{0}^{\infty} f(\mathbf{x}) d \mathbf{x}
\end{aligned}
$$

and with $\epsilon_{0}=0$ and $l_{n}^{\prime} s=0$, then inequality (3.2) reads

$$
\begin{aligned}
& \int_{0}^{\infty} \cdots \int_{0}^{\infty} \exp \left(x_{1} \cdots x_{n} \int_{x_{1}}^{\infty} \cdots \int_{x_{n}}^{\infty} \ln f(\mathbf{y})\left(\prod_{i=1}^{n} y_{i}^{2}\right)^{-1} d \mathbf{y}\right) d \mathbf{x} \\
& \leq e^{-n} \int_{0}^{\infty} \cdots \int_{0}^{\infty} f(\mathbf{x}) d \mathbf{x} .
\end{aligned}
$$

Both of the constants $e^{n}$ and $e^{-n}$ are sharp.

Remark 3.3. For the case $n=1$, (3.8) coincides with the classical Pólya-Knopp inequality (see [12])

$$
\int_{0}^{\infty} \exp \left(\frac{1}{x} \int_{0}^{x} \ln f(y) d y\right) d x \leq \mathbf{e} \int_{0}^{\infty} f(x) d x
$$

and (3.9) corresponds to the inequality

$$
\int_{0}^{\infty}\left(\exp x \int_{x}^{\infty} \ln f(\mathbf{y}) \frac{1}{y^{2}} d \mathbf{y}\right) d x \leq \mathbf{e}^{-1} \int_{0}^{\infty} f(x) d x
$$

Example 3.4. Another important special case of Theorem 3.1 is obtained by choosing $\epsilon=-1$ and $l_{i}^{\prime} s=\infty$ in (3.1) and $\epsilon_{0}=-1$ and all $l_{i}^{\prime} s=0$ in (3.2). Then we obtain the following equivalent and sharp Pólya-Knopp-type inequalities:

$$
\begin{aligned}
& \int_{0}^{\infty} \cdots \int_{0}^{\infty} \exp \left(\frac{1}{x_{1} \cdots x_{n}} \int_{0}^{x_{1}} \cdots \int_{0}^{x_{n}} \ln f(\mathbf{y}) d \mathbf{y}\right)\left(\prod_{i=1}^{n} x_{i}\right)^{-1} d \mathbf{x} \\
& \leq \int_{0}^{\infty} \cdots \int_{0}^{\infty} f(\mathbf{x})\left(\prod_{i=1}^{n} x_{i}\right)^{-1} d \mathbf{x}
\end{aligned}
$$

and

$$
\begin{aligned}
& \int_{0}^{\infty} \cdots \int_{0}^{\infty} \exp \left(x_{1} \cdots x_{n} \int_{x_{1}}^{\infty} \cdots \int_{x_{n}}^{\infty} \ln f(\mathbf{y})\left(\prod_{i=1}^{n} y_{i}^{2}\right)^{-1} d \mathbf{y}\right)\left(\prod_{i=1}^{n} x_{i}\right)^{-1} d \mathbf{x} \\
& \leq \int_{0}^{\infty} \cdots \int_{0}^{\infty} f(\mathbf{x})\left(\prod_{i=1}^{n} x_{i}\right)^{-1} d \mathbf{x} .
\end{aligned}
$$

Remark 3.5. We believe that the information in Example 3.4 is new also for the case $n=1$. Moreover, (3.10) may be regarded as a limit case of (2.1) when all 
$l_{i}^{\prime} s=\infty$ and (3.11) may be regarded as the limit case of the corresponding dual version of (2.1).

Remark 3.6. The inequality (3.2) can also be proved directly by using (2.5) and discussing as in the proof of (3.1). In fact, by replacing $f(\mathbf{x})$ by $(f(\mathbf{x}))^{\frac{1}{p}} \prod_{i=1}^{n} x_{i}^{2}$ in (2.5) we find that (note that $\epsilon_{0}=2 p-\epsilon-2$ )

$$
\begin{aligned}
& \int_{l_{1}}^{\infty} \cdots \int_{l_{n}}^{\infty}\left(x_{1} \cdots x_{n}\right)\left(\int_{x_{1}}^{\infty} \cdots \int_{x_{n}}^{\infty}(f(\mathbf{y}))^{\frac{1}{p}}\left(\prod_{i=1}^{n} y_{i}^{2}\right)^{-1} d \mathbf{y}\right)^{p} \prod_{i=1}^{n} x_{i}^{-\epsilon-2} d \mathbf{x} \\
& \leq\left(\frac{p}{\epsilon_{0}+1-p}\right)^{n p} \int_{l_{1}}^{\infty} \cdots \int_{l_{n}}^{\infty} f(\mathbf{x}) \prod_{i=1}^{n}\left(1-\left(\frac{l_{i}}{x_{i}}\right)^{\frac{\epsilon_{0}+1-p}{p}}\right) \mathbf{x}^{-\epsilon-2} d \mathbf{x} .
\end{aligned}
$$

Now we let $p \rightarrow \infty$ and note that then

$$
\begin{gathered}
\left(\frac{p}{\epsilon_{0}+1-p}\right)^{n p}=\left(\frac{p}{p-1-\epsilon}\right)^{n p} \rightarrow e^{(1+\epsilon) n} \\
\frac{\epsilon_{0}+1-p}{p}=\frac{p-\epsilon-1}{p} \rightarrow 1,
\end{gathered}
$$

and

$$
\begin{aligned}
& \left(x_{1} \cdots x_{n} \int_{x_{1}}^{\infty} \cdots \int_{x_{n}}^{\infty}(f(\mathbf{y}))^{\frac{1}{p}}\left(\prod_{i=1}^{n} y_{i}^{2}\right)^{-1} d \mathbf{y}\right)^{p} \\
& \rightarrow \exp \left[x_{1} \cdots x_{n} \int_{x_{1}}^{\infty} \cdots \int_{x_{n}}^{\infty} \exp f(\mathbf{y})\left(\prod_{i=1}^{n} y_{i}^{2}\right)^{-1} d \mathbf{y}\right] .
\end{aligned}
$$

(The scale of Power means $\mathcal{P}_{\alpha}, \alpha=\frac{1}{p}$, of the function $f(\mathbf{y})$ over the set $\left(x_{1}, \infty\right) \times$ $\left(x_{2}, \infty\right) \times \cdots \times\left(x_{x}, \infty\right)$ with measure $x_{1} \cdots x_{n}\left(\prod_{i=1}^{n} y_{i}^{2}\right)^{-1} d \mathbf{y}$ converges to the geometric mean $\mathcal{P}_{0}$ when $\alpha \rightarrow 0+$ ). By now replacing $-\epsilon-2$ by $\epsilon_{0}$ and combining (3.12)-(3.15) we obtain (3.2).

\section{ACKnowledgements.}

The first author expresses his gratitute to the Abdus Salam International Centre for Theoretical Physics, Trieste, Italy for financial support to carry out this research within the framework of the Associateship scheme of the centre. All authors express their gratitude to the careful referee whose advices improved the final version of this paper. 


\section{REFERENCES}

1. E.K. Godunova, Inequalities based on convex functions, Izv. Vysh. Uchebn. Zaved. Matematika 47 (1965), no. 4, 45-53; English transl. in Amer. Math. Soc., Transl., II Ser. 88 (1970), 57-66.

2. E.K. Godunova, Integral inequalities with convex functions, Izv. Vysh. Uchebn. Zaved. Matematika 68 (1968), 47-49 (Russian).

3. G.H. Hardy, Note on a theorem of Hilbert, Math. Z. 6 (1920), 314-317

4. G.H. Hardy, Notes on some points in the integral calculus, LX. An inequality between integrals, Messenger of Math. 54 (1925), 150-156.

5. G.H. Hardy, Notes on some points in the integral calculus. LXIV. Further inequalities between integrals, Messenger of Math. 59 (1928), 12-16.

6. G.H. Hardy, J.E. Littlewood and G. Pólya, Inequalities, Camlridge University Press, Camlridge, 1959.

7. C.O. Imoru, On some integral inequalities related to Hardy's, Canad. Math. Bull. 20 (1977), no. 3, 307-312.

8. J.L.W.V. Jensen, Om konvexe funktioner og uligheter mellom middelvaerdier, Nyt. Tidsskr. Math. 16B (1905), 49-69.

9. J.L.W.V. Jensen, Sur les fonctions convexes et les inegalities entre les valeurs moyennes, Acta Math. 30 (1906), 175-193.

10. S. Kaijser, L. Nikolova, L.-E. Persson and A. Wedestig, Hardy type inequalities via convexity, Math. Inequal. Appl. 8 (2005), no. 3, 403-417.

11. S. Kaijser, L-E. Persson and A. Öberg, On Carleman and Knopp's inequalities, J. Approx. Theory 117 (2002), 140-151.

12. K. Knopp, Über Reihen mit positiven Gliedern, J. London Math. Soc. 3 (1928), 205-212.

13. K. Krulić, Generalizations and refinements of Hardy's inequalities, PhD. Thesis, Department of Mathematics, University of Zagreb (2010).

14. K. Krulić, J. Pečarić and L.-E. Persson, Some new Hardy-type inequalities with general kernels, J. Math. Inequal. 12 (2009), no. 3, 473-485.

15. A. Kufner, L. Maligranda and L.-E. Persson, The prehistory of the Hardy inequality, Amer. Math. Monthly 113 (2006), 715-732.

16. A. Kufner, L. Maligranda and L.-E. Persson, The Hardy Inequality - About its History and Some Related Results, Vydavatelsky Servis Publishing House, Pilsen, 2007.

17. J.A. Oguntuase, C.A. Okpoti, L.-E. Persson and F.K.A. Allotey, Weighted multidimensional Hardy type inequalities via Jensen's inequality, Proc. A. Razdmadze Math. Inst. 144 (2007), 107-118.

18. J.A. Oguntuase, C.A. Okpoti, L.-E. Persson and F.K.A. Allotey, Multidimensional Hardy type inequalities for $p<0$ and $0<p<1$, J. Math. Inequal. 1 (2007), 1-11.

19. J.A. Oguntuase and L.-E. Persson, Hardy type inequalities via convexity - the journey so far, Austral. J. Math. Anal. Appl. 7 (2010), no. 2, Art. 18, 19 pp.

20. J.A. Oguntuase, L.-E. Persson and A. Čižmešija, Multidimensional Hardy-type inequalities via convexity, Bull. Austral. Math. Soc. 77 (2008), 245-260.

21. J.A. Oguntuase, L.-E. Persson and E.K. Essel, Multidimensional Hardy-type inequalities with general kernels, J. Math. Anal. Appl. 348 (2008), 411-418.

22. J.A. Oguntuase, L.-E. Persson, E.K. Essel, B.A. Popoola, Refined multidimensional Hardytype inequalities via superquadracity, Banach J. Math. Anal. 2 (2008), no. 2, 129-139.

23. L.-E. Persson and J.A. Oguntuase, Refinement of Hardy's inequality for "all" $p$, K. Mikio and L. Maligranda (eds.), Proc. Inter. Symp.lanach Func. Spaces (ISlFS 2006), Kitakyushu, Japan, Septemler 14-17, 2006. Yokohama: Yokohama Pullishers. 129-144 (2008).

24. L.-E. Persson and N. Samko, What should have happened if Hardy had discovered this?, J. Inequal. Appl. 2012, 2012:29, 11 pp. 
1 Department of Mathematics, Federal University of Agriculture, P.M.B. 2240, Abeokuta, Ogun State, Nigeria.

E-mail address: oguntuase@yahoo.com

E-mail address: sonubi03@yahoo.com

2 Department of Mathematics, Lule̊ University of Technology, SE-971 87 LuleÅ, Sweden; Narvik University College, P.O. Box 385, N-8505 Narvik, Norway.

E-mail address: Lars-Erik.Persson@ltu.se

E-mail address: Natasha.Samko@ltu.se 